\title{
POTENCIAL ESCÉNICO DE LA RESERVA NATURAL CERRO DATANLÍ-EL DIABLO EN JINOTEGA, CON FINES ECO TURÍSTICOS
}

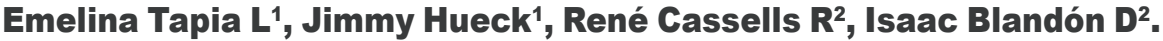

${ }^{1}$ Ing.Agrónomo especialista en Forestal e Ing. Agrónomo especialista en Manejo de los Recursos Naturales. Docentes DMByE, FARENA/UNAApdo. 453

${ }^{2}$ Graduados Ingeniería en RRNN e Ingeniería Forestal, FARENA/UNA.

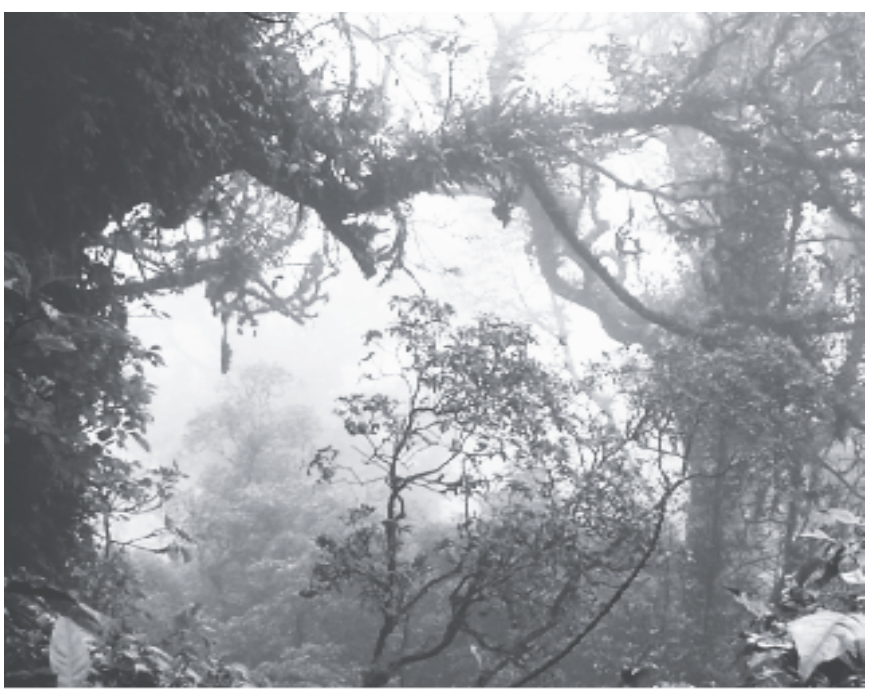

\section{RESUMEN}

El estudio fue realizado en la RN Cerro Datanlí- El Diablo, Jinotega. El objetivo principal fue identificar sitios con potencial belleza escénica aun no considerados para la ejecución de actividades ecoturísticas que tiendan a mejorar la calidad de vida de sus pobladores locales a partir de la visita de turistas y nacionales. Los mejores atractivos naturales e infraestructura existente se homogenizaron mediante la consulta a lugareños y a través de la observación personal. La metodología se desarrolló en tres etapas: (1) Recolección de la información secundaria, selección y ubicación de la zona de estudio y de contactos (MARENA, PAGJINO, Líderes locales). (2) Giras de campo para llevar a cabo: el DRP auxiliado con tres herramientas participativas; la identificación de 22 sitios con potencial ecoturístico, 3 problemas principales que limitan su desarrollo, recorridos por 7 senderos ecológicos que facilitó la identificación de 17 sitios posibles como miradores de paisajes diversos y 5 sitios donde se pueden observar y escuchar cantar aves exóticas como el Quetzal; aplicación de encuestas a los pobladores locales (niños y jóvenes ambientalistas, mujeres y productores) con el fin de conocer su participación en actividades de protección, conservación y manejo de los recursos naturales. (3) Elaborar los mapas de los senderos a través del programa Arc View 3.2, se ubicaron los principales atractivos, propuestas de cambios en los recorridos basados en pendientes del terreno, análisis de visibilidad de miradores y

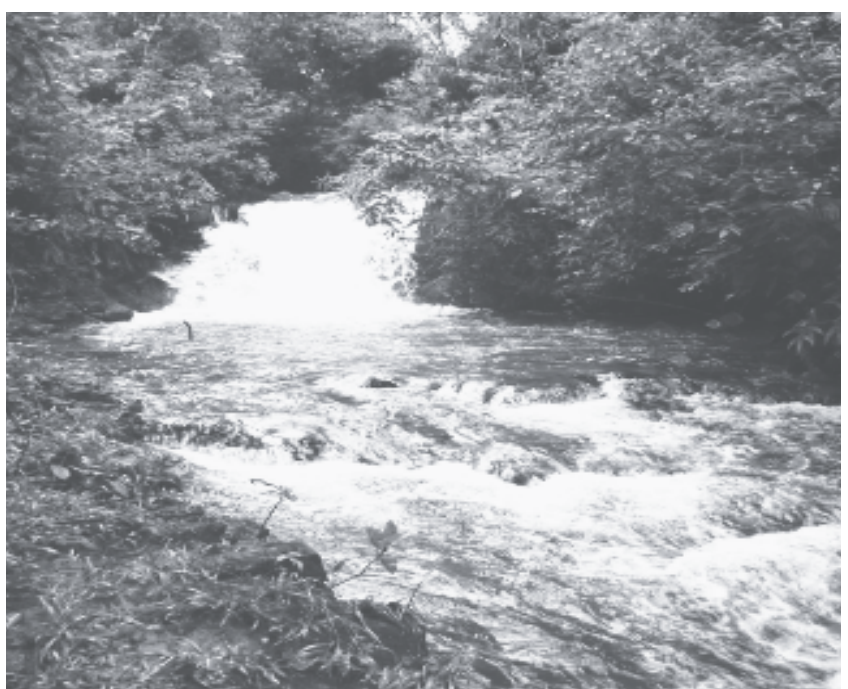

\section{ABSTRACT}

This study was carried out in the Natural Reserve Cerro "Datanlí-El Diablo, Jinotega. The purpose was to identify places with potential scenic beauty not yet considered for ecotourism activities. The manly idea is to change a common life of de local people that live there for the traffic of visitors. Through a participatory consult to the villagers and personal observations were recognized the natural attractiveness and existent infrastructure in the protected area. The methodology was developed in three phases: (1) the gathering of secondary information, selection and location of the zone of study and establishment of the contacts (MARENA, PAGJINO and local people). (2) Field trips: a PRA(Participatory Rural Appraisal), three participative tools used; also, the identification of 22 places with ecotourism potential and the three problems that limit their development. A survey of the seven trails in the studied area performed, identifying a total of 17 places that could serve of landscape viewers and 5 places to observe and listening exotic birds like the Quetzal. Applications of surveys to the local residents (children and young environmentalist, women and producers) with the purpose of knowing their participation in the activities of protection, conservation and management of natural resources in the protected area. (3) Assisted for the program Arc View 3.2, maps of the trails and these show the walking trails and their main attractive elaborated; it is proposed some changes to the route, based on 
según los atractivos principales en los sendero. La información facilitó incluir el diseño de un modelo de infraestructura en los senderos (gradas, miradores y puentes) para el tráfico de visitantes y la elaboración de tres propuestas de paquetes turísticos que contienen información básica de los recursos naturales existentes en la reserva natural.

Palabras clave: Atractivos naturales, calidad de vida, Diagnóstico Rural Participativo, ecoturismo, encuestas, miradores de senderos, paquetes turísticos, potencial belleza escénica, Sistema de Posicionamiento Global GPS. the steepness of the slopes, the visibility of the viewers and according to the main attractiveness of each walking trail. All the information was included to elaborate the infrastructure design (steps, viewers and bridges) for the traffic of visitors and was elaborated three proposals of tourist packages that contain basic information of the natural existent resources in the natural reserve.

Keywords: Ecotourism, Global Positional System (GPS), landscape viewers, natural attractive, Participatory Rural Appraisal (PRA), Potential scenic beauty, quality life condition, surveys to the local residents, tourist packages, visibility of the viewers, walking trails.
$\mathrm{E}$ 1 Ministerio del Ambiente y los Recursos Naturales (MARENA) ha promovido la creación de condiciones que conlleven al desarrollo del ecoturismo en áreas protegidas de nuestro país, mediante la promoción de los atractivos turísticos, así como la contemplación de la belleza escénica presente en éstas áreas; también incluye la promoción del desarrollo de facilidades para la recreación y el turismo, como parte de la gestión ambiental y el aprovechamiento de los servicios ambientales que generan los espacios naturales (MARENA, 2004). Sin embargo, los recursos naturales suelo, agua y diversidad de flora y fauna que se pueden apreciar en estos ecosistemas, se encuentran en condiciones de degradación, fragmentados y perturbados por actividades cafetalera $u$ otras que indican la mala utilización y por el poco conocimiento que se tiene sobre sus riquezas y formas de aprovechamiento.

La Reserva Natural Datanlí-El Diablo, localizada en el Departamento de Jinotega, es un área protegida que representa uno de los pocos lugares dentro del territorio nicaragüense que conserva una gran diversidad biológica de especies consideradas en peligro de extinción. Se caracteriza, por la existencia de especies típicas de un bosque tropical nuboso, por los valores económicos que representan los recursos hídricos y productivos y por la importancia sociocultural y ambiental de su entorno (MARENA, et al, 2002). El aprovechamiento sostenible de los recursos naturales con que cuenta esta área protegida, incluyendo su belleza escénica, representa una alternativa económica importante para mejorar la calidad de vida de sus habitantes (MARENA, 2002; PAGJINO, 2003).

La finalidad de ésta investigación, llevada a cabo en un lapso de 9 meses, fue por lo tanto, el poder determinar los sitios de mayor belleza o potencial escénico presentes en los senderos propuestos por el comité de manejo de la reserva natural Cerro Datanlí-El Diablo con fines ecoturísticos. La información generada y analizada en este estudio es de gran relevancia para la planificación de futuras actividades turísticas recogidas en el plan de manejo de la reserva, lo que a su vez podría mejorar la calidad de vida de sus pobladores a través de los servicios que genere y los aportes económicos brindados por los visitantes nacionales y extranjeros interesados en apreciar sus bellezas, pero todo bajo el marco de manejo sostenido y participativo que garanticen la prolongación de la diversidad biológica que posee.

\section{MATERIALES Y MÉTODOS}

Ubicación Geográfica. Reserva Natural Cerro Datanlí - El Diablo está localizada en el departamento de Jinotega en el Norte de Nicaragua (Figura 1) y cuenta con una extensión territorial de 5,848.94 hectáreas $(58.4794 \mathrm{Km} 2)$. Sus límites presentes son de tres tipos: límites naturales (ríos y quebradas), límites construidos por el hombre (carreteras, caminos y trochas) y límites cartográficos (curvas de nivel). Teniendo su ubicación geográfica en un cuadrante con las siguientes coordenadas: $13^{\circ} 04^{\prime} 42.1^{\prime \prime}$ y $13^{\circ} 10^{\prime} 10.7^{\prime \prime}$ Latitud Norte y $85^{\circ} 49^{\prime} 49.4$ " y $85^{\circ} 54^{\prime} 58.6$ " de Longitud Oeste (MARENA, 2002).

Condiciones Climáticas. Este valioso ecosistema de nebliselva presenta una precipitación promedio entre los 1,650 mm en la parte Norte de la Reserva, y 2,600 mm anuales en la parte Sur. De acuerdo con MARENA (2002), presenta temperaturas variadas: en las partes bajas, a 1,000 msnm, la temperatura promedio es de $20^{\circ} \mathrm{C}$; en cambio en las partes altas, a 1,600 msnm, la temperatura promedio es de $12.5^{\circ} \mathrm{C}$. Los meses más calurosos son Abril y Mayo y los meses más fríos Diciembre y Febrero.

Características topográficas. Forma parte de un elevado macizo montañoso, cuyo relieve predominante son altas montañas, colinas escarpadas, planicies secciona- 
das, depresiones entre cordilleras y valles encajonados; aunados a las características propias de las formaciones geológicas de los suelos, conforman una de las zonas más productivas del país incidiendo en este aspecto los diversos grados de precipitación y humedad existente en las diversas zonas de la región. Sus alturas varían desde los $900 \mathrm{msnm}$ (metros sobre el nivel del mar) a nivel de la quebrada de La Bastilla hasta la cumbre del Cerro El Diablo a 1,650 msnm. La altitud promedio es cercana a los 1,300 msnm. Al interior de la reserva se encuentran ocho cumbres mayores de $1,350 \mathrm{msnm}$.

Proceso metodológico. Se establecieron tres etapas metodológicas que permitieron la integración conjunta de todos los elementos necesarios para abordar de forma real y directa los objetivos planteados en el estudio (Figura 2).

Diagnóstico Rural Participativo (DRP). El taller de planificación se realizó en MARENA Jinotega,

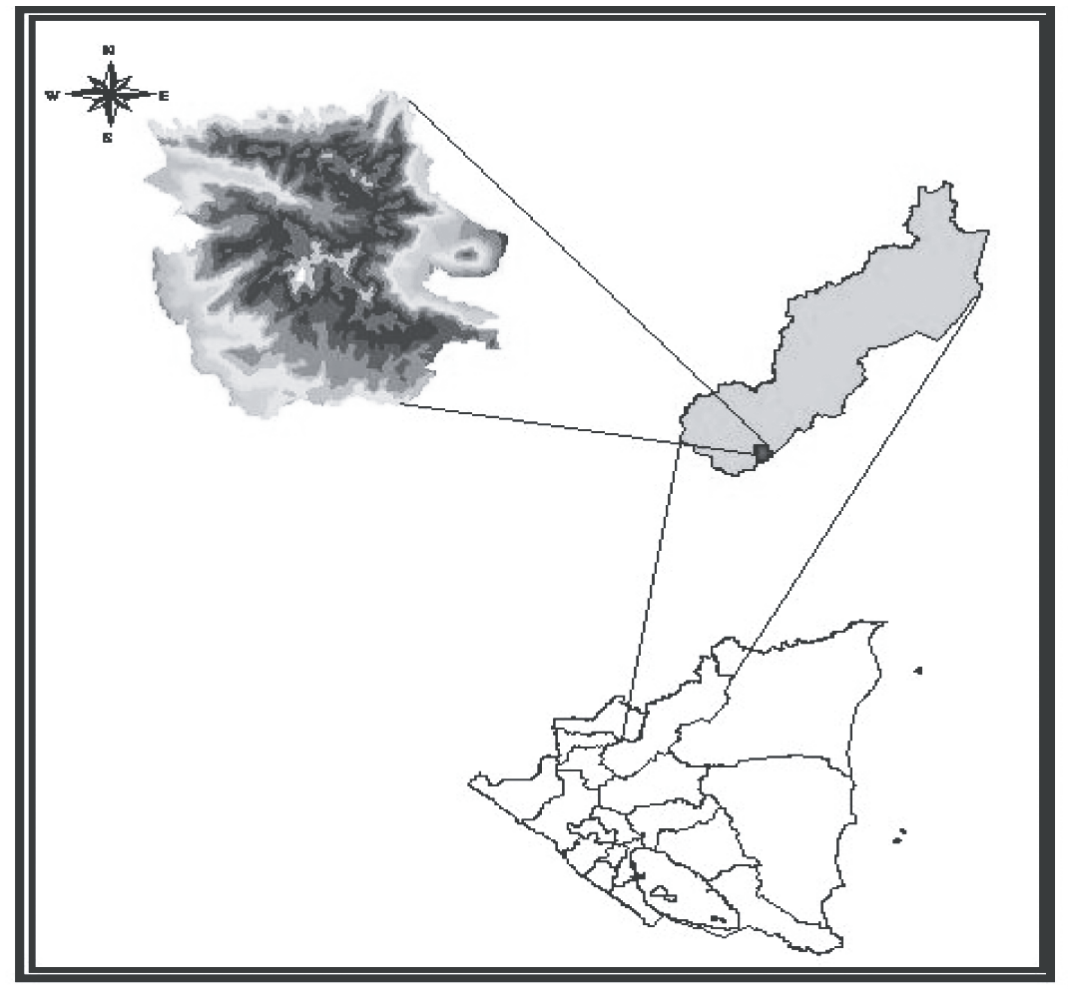

MAPA DE UEICACION DE LA

RE SERVA NATURAL CERRO

D AT AN LÍ-EL DIABLO

LEYEND A

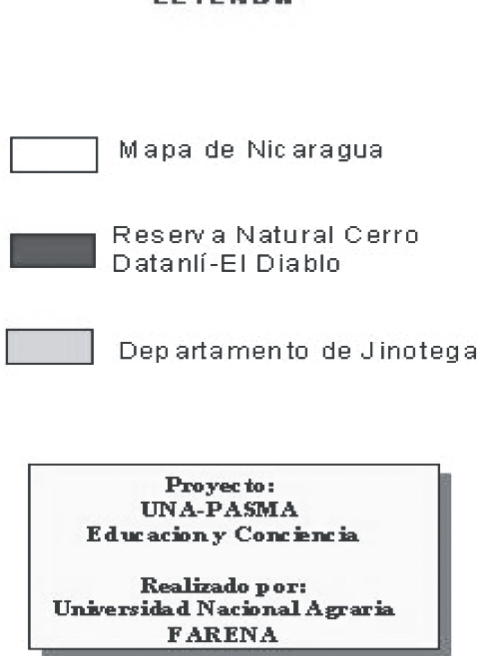

Figura 1. Mapa de ubicación de la Reserva Natural Cerro Datanlí-El Diablo. Jinotega, Nicaragua.

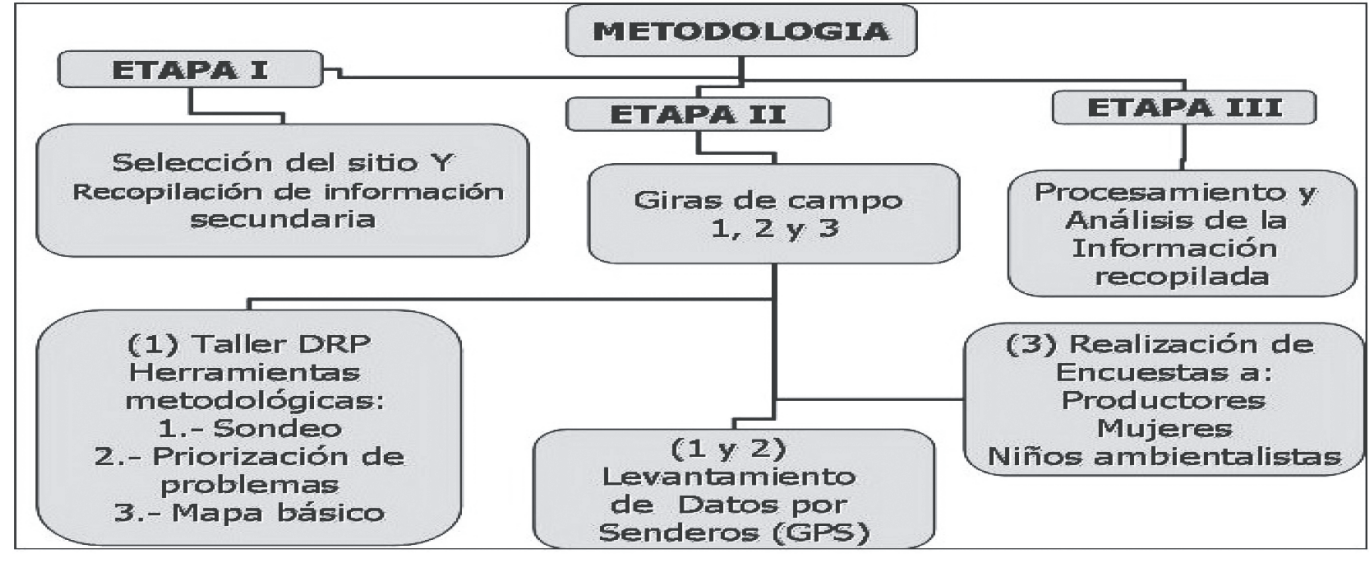

Figura 2. Metodología implementada en la realización del estudio del potencial escénico de la Reserva Natural Cerro Datanlí-El Diablo con fines ecoturísticos. Jinotega, Nicaragua. 
participaron miembros del comité de manejo de la reserva, en conjunto con dos investigadores y dos estudiantes de la Universidad Nacional Agraria para determinar primeramente que los cerros "El Gobiado" (Datanlí) en la Cooperativa Lina Herrera (zona Norte) y "El Cerro el Diablo", cerca de la Comunidad La Esmeralda (Zona Sur), eran los lugares más apropiados para la realización del estudio. Estos lugares presentan la mayor altitud y cobertura boscosa y además, albergan una gran cantidad de especies de Flora y Fauna Silvestre y ofrecen un clima frió de montaña con hermosas vistas panorámicas que presentan gran valor ecológico y un gran potencial eco turístico. En el taller se desarrollaron tres herramientas participativas: a. La prueba rápida diagnóstico de conocimientos, permitió la armonización de algunos conceptos relacionados al ecoturismo tales como: Belleza, turismo, ecoturismo, servicios, bienes y recursos.

b. El mapa básico participativo de la reserva natural, se realizó con la finalidad de obtener información referente a los posibles sitios de interés turístico, representados por códigos, los cuales fueron ubicados en un mapa de la reserva distribuido en cuatro cuadrantes. Además, fue posible determinar con los miembros del comité de manejo en conjunto con MARENA los senderos propuestos a ser recorridos en los días subsiguientes a la investigación (Figura 3).

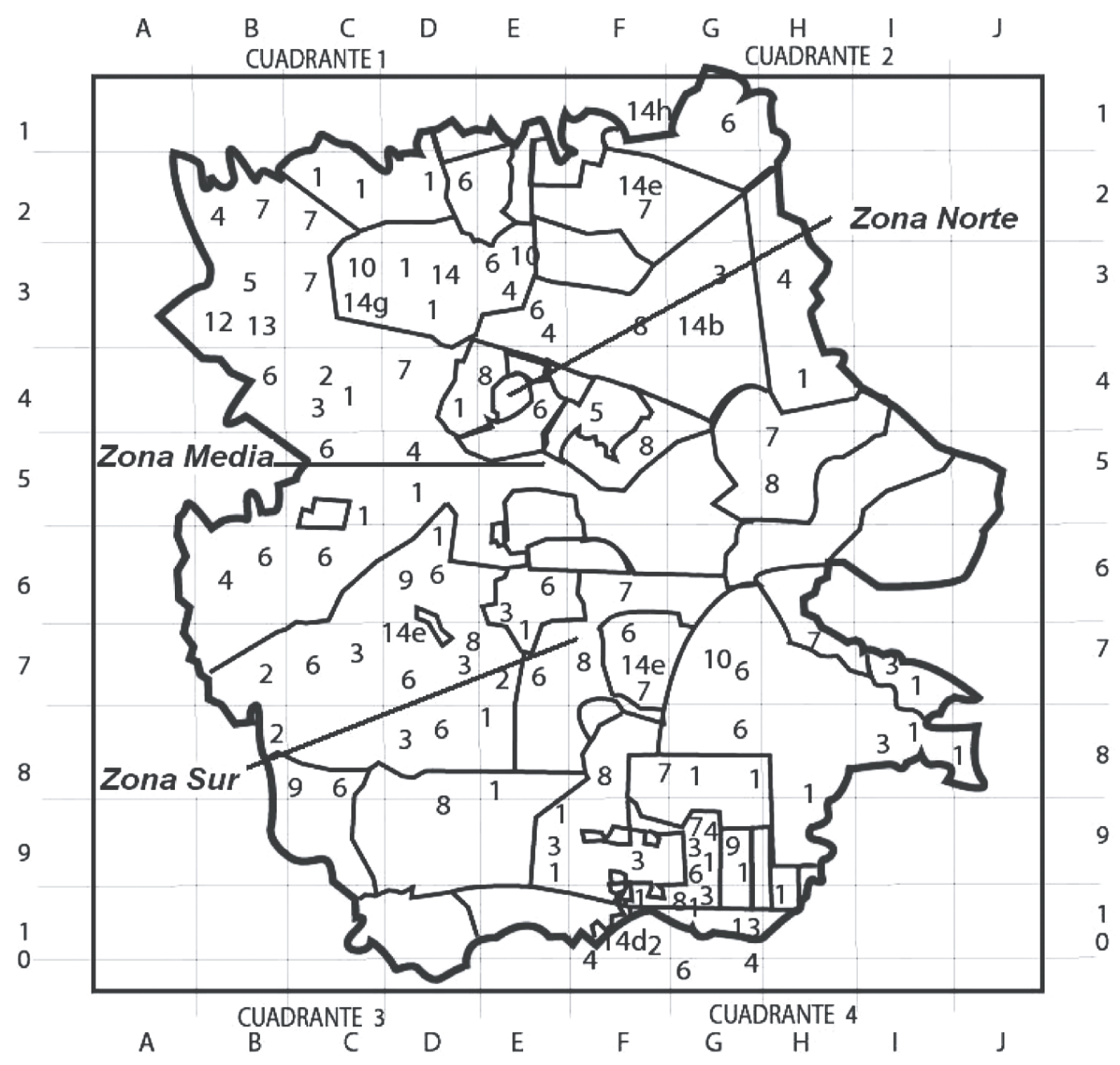

Figura 3. Mapa básico participativo de la Reserva Natural Cerro "Datanlí - El Diablo". Jinotega, Nicaragua. 
c. La Matriz de Priorización de problemas permitió la descripción de las principales limitantes que podrían obstaculizar el desarrollo del Ecoturismo en la Reserva y el orden, de acuerdo a su percepción, en que estos debían ser atendidos.

Georeferenciación de puntos de interés. Con el uso del GPS (Sistema de Posicionamiento Global) se logró obtener el levantamiento de las poligonales de los siete senderos y adicionalmente se marcaron sitios donde se producen Orquídeas (Orquidiarios) y dos saltos de agua localizados en los límites de la reserva natural: "Salto La Bujona" de aproximadamente $12 \mathrm{~m}$ y "Salto Las Nubes" con una altura de 3-4 m. Así mismo, se tomaron los puntos de interés como miradores de aves y tramos del trayecto en donde se puede escuchar cantar y ver a las aves "El Rancho" y "El Jilguero, sitios de descanso, lugares donde se pueden apreciar orquídeas, miradores de paisajes y vistas diversas hacia el Lago de Apanas y árboles de Roble encino de considerable tamaño. Además, durante la caminata se recopiló información relacionada con sitios de interés turístico, leyendas, sitios históricos, quebradas, ojos de agua, formaciones rocosas, plantas medicinales, posibles miradores de escenas paisajísticas con diversidades de intereses, entre otros.

Encuestas y entrevistas. Las encuestas fueron aplicadas a 44 personas (16 productores, 13 mujeres y 15 niños y

Tabla 1. Variable A- Focos locales: Productores-Mujeres. Reserva Natural Cerro Datanlí-El Diablo. Jinotega, Nicaragua

I.- Ámbito: $\quad$ Reserva Natural: Preservar, Conservar y Rescatar el Medio ambiente, los recursos naturales y los valores culturales

Parámetro 1. Percepción Comunitaria. Problemas vs Potencialidades en la RN

Parámetro 2: $\quad$ Perfil Ecoturístico // Plan y Programa del Manejo del área

Promoción de Turismo Rural vs Los Recursos existentes en la RN

Parámetro 3: $\quad$ Promoción de Servicios y Conservación

Criterio 1: Con la promoción de servicios, se mantienen y aumentan los beneficios

económicos de los pobladores locales

Criterio 2: El establecimiento y mantenimiento de los senderos promueve la integración

comunitaria

Criterio 3: El manejo Sostenible y la conservación de los RRNN se logra a través de la

participación conjunta en actividades de recreación, Educación Ambiental y en otros indicadores afines

Tabla 2. Variable B- Focos locales: Niños y Jóvenes ambientalistas. Reserva Natural Cerro Datanlí-El Diablo. Jinotega, Nicaragua

I.- Ámbito: $\quad$ Reserva Natural: Preservar, Conservar y Rescatar el Medio ambiente, los recursos naturales y los valores culturales

Parámetro 1: $\quad$ Percepción de niños y jóvenes que viven en la RN. Valoración del potencial de la Reserva Natural vs su Participación en actividades de conservación de los RRNN

Criterio 1.- Valoración y conocimiento

El grado de conocimiento y valoración de los recursos existentes en la RN por parte de los niños y jóvenes de la comunidad se logra a través de su integración en las diferentes actividades que realizan sus familiares y el comité local dentro de la $\mathrm{RN}$ Criterio 2. Participación y concientización El grado de participación y concientización de los niños y jóvenes de la comunidad se logra involucrándoles en las diferentes actividades relacionadas con el manejo y potencial de los RRNN existentes en la RN 
jóvenes) durante 3 días y fueron estructuradas a partir del modelo "Herramienta de Ecología de paisajes y la calidad de Bosques, Principios, criterios, indicadores y verificadores (PCI\&V)" de Lammerts y Blom (1997) en GTZ-BMZ-UICN-WWF del año 1999 y se incluyeron aspectos del Plan de Manejo. Los aspectos considerados para el ámbito, los parámetros y criterios se establecieron en dos grandes grupos definidos como variables o focos locales tales como variable A: productores y mujeres (Tabla 1) y variable B: niños y jóvenes ambientalistas (Tabla 2).

\section{RESULTADOS Y DISCUSIÓN}

Prueba rápida diagnóstico de conocimientos. Las definiciones de Belleza, Turismo, Ecoturismo, Servicios, Bienes y Recursos que definieron los productores, fueron relacionadas con el potencial escénico de la Reserva (Tabla 3).

Mapa básico participativo y claves numéricas. los productores ubicaron un total 22 principales atractivos turísticos con que cuenta la reserva. 14 lugares de éstos destacan aspectos de ecología de paisajes que se encuentran dentro y fuera de los senderos y 8 sitios de valor histórico y de leyendas que consideran tiene la reserva. Esta información consensuada entre la zona Norte y Sur (Tabla 4).

Matriz de priorización de problemas. se detectó que la ausencia de programas o proyectos que involucren a los pobladores de la comunidad en capacitaciones y/o talleres relacionados con el Pago por Servicios Ambientales, era el principal problema a resolver de forma inmediata, seguido por la falta de Reparación y Construcción de Caminos de acceso en la reserva, ya que éstos solo se encuentran disponibles en el verano y el tercer aspecto a considerar es que los Organismos e Instituciones no cuentan con un plan continuo de promoción de asistencia técnica y aportes de conocimientos para el manejo sostenible de la reserva. Cabe mencionar que la ausencia de un Plan de Desarrollo turístico, energía eléctrica y la Telefonía comunitaria, resultaron como problemas menos priorizados pero si debían ser resueltos.

Senderos de la zona Norte y Sur de la RN. En la zona Norte y Sur se determinaron las coordenadas de puntos continuos de un total de siete senderos representativos

Tabla 3. Herramienta Prueba Rápida Diagnóstico de Conocimientos realizada en la Reserva Natural Cerro "Datanlí - El Diablo". Jinotega, Nicaragua. 2004

\begin{tabular}{ll}
\hline Conceptos & Definiciones brindadas por los productores \\
\hline Belleza & Canto del mono congo, de pájaros y quetzales. El entorno agradable a los sentidos del hombre, \\
& la biodiversidad, montañas de nebliselva, sentir, oler, mirar, escuchar. La vista del Lago de \\
& Apanás. Apreciar los árboles de Canelo, Posan y María que producen alimento y es hospedero \\
& de animales (tucanes, ardillas, sargento, chiltote, oropéndola, sahino, guardatinaja y venados). \\
& Ver como se alimentan los animales. \\
& Significa dinero, salir de la rutina, salud mental, física y emocional. Hay diferentes tipos de \\
Turismo & turismo: mochileros, turistas que viajan de lugar en lugar y de casa en casa con el objetivo de \\
& pasear. Viajar para salir de la rutina, salir para distraerse visitando lugares bonitos. \\
Ecoturismo & Viajar para relacionarse con la naturaleza. Convivir en un ambiente de montaña, ver los \\
& animales y las plantas silvestres pero tratando de no destruir la naturaleza. \\
Servicios & Infraestructura, la finca, el ganado, la flora, la fauna, los ríos, corrientes de agua, lagos, bosque \\
de nebliselva. & Vender comida, brindar alojamiento, un servicio es la belleza de la reserva, los miradores, las \\
Bienes & artesanías, leyendas, disfrutar de la naturaleza, belleza escénica, ofrecer agua de la montaña. \\
Recursos & Son los bienes materiales con los que contamos hoy. Recursos con los que podríamos contar \\
& (recursos potenciales).
\end{tabular}


Tabla 4. Herramienta Mapa Básico Participativo de la Reserva Natural Cerro “Datanlí - El Diablo”. Jinotega, 2004

Principales Atractivos Turísticos detectados en la $14 \quad$ Sitios de Valor Histórico
zona Norte y Sur de la Reserva.

\begin{tabular}{llll}
\hline Lugares & Nombre del sitio & Lugares & Valor histórico y leyendas \\
\hline 01 & Ojo de agua & $14 \mathrm{~d}$ & Cementerio \\
02 & Ríos & $14 \mathrm{e} 1$ & Leyenda del príncipe del Gobiado \\
03 & Quebradas & $14 \mathrm{e} 2$ & Leyenda del Gobiado \\
04 & Caídas de agua o Cascadas & $14 \mathrm{e} 3$ & Leyenda del Cerro el Diablo \\
05 & Lagunas (lagunetas) & $14 \mathrm{e} 4$ & batallas históricas donde pasó Sandino \\
06 & Miradores & $14 \mathrm{e} 5$ & Batallas históricas explosión de minas \\
07 & Plantas raras, únicas y exóticas. & $14 \mathrm{f}$ & Deslizamientos de tierra. \\
08 & Animales raros, únicos, exóticos & $14 \mathrm{~g}$ & Sepultura en la Fila del Gobiado \\
& (ave quetzal y Rancho entre otros), & & \\
09 & Plantas medicinales. & & \\
10 & Cuevas. & \\
11 & Orquidiarios & \\
12 & formaciones rocosas o peñascos: & \\
& (Piedra del amor y Peña del Gobiado) & \\
13 & Artesanías. &
\end{tabular}

Tabla 5. Senderos representativos encontrados en la RN Cerro "Datanlí-El Diablo. Jinotega

\begin{tabular}{lll}
\hline $\begin{array}{l}\text { Nombre } \\
\text { Sendero }\end{array}$ & $\begin{array}{l}\text { Longitud } \\
\text { y pendiente }\end{array}$ & Flora y Fauna \\
\hline Zona Norte: Cerro El Gobiado & \\
\hline El Congo & $\begin{array}{l}3.66 \mathrm{~km} . \\
0-50^{\circ}\end{array}$ & $\begin{array}{l}\text { Es el de mayor recorrido en la zona, con pendientes fuertes y gran } \\
\text { variedad de plantas y animales principalmente monos Congo y el } \\
\text { ave Quetzal. }\end{array}$ \\
El León & $3.14 \mathrm{~km}$. & $\begin{array}{l}\text { Pendientes moderadas, con abundantes ojos de agua, quebradas, } \\
\text { plantas ornamentales, medicinales y fauna variada } \\
\text { Es el de menor recorrido, con pendientes suaves, abundantes } \\
\text { árboles de Roble enzino, matapalos y aves exóticas. }\end{array}$
\end{tabular}

Zona Sur: Cerro el Diablo

\begin{tabular}{|c|c|c|}
\hline La Mocuana & $\begin{array}{l}0.4 \mathrm{~km} . \\
0-40^{\circ}\end{array}$ & $\begin{array}{l}\text { Es el de menor recorrido, con un pequeño humedal y plantas } \\
\text { Ornamentales y vistas hermosas hacia el Cerro El Volcán. }\end{array}$ \\
\hline La Pedrera & $\begin{array}{l}2.14 \mathrm{~km} . \\
0-45^{\circ}\end{array}$ & $\begin{array}{l}\text { Encontramos ojos de agua, quebradas presencia de insectos, } \\
\text { moluscos y abundante vegetación. }\end{array}$ \\
\hline El Quetzal & $\begin{array}{l}3.52 \mathrm{~km} \\
0-50^{\circ}\end{array}$ & $\begin{array}{l}\text { Encontrar presencia de moluscos, reptiles, anfibios y al atractivo } \\
\text { ave Quetzal. }\end{array}$ \\
\hline El Diablo & $\begin{array}{l}5.26 \mathrm{~km} \\
0-50^{\circ}\end{array}$ & $\begin{array}{l}\text { Es el de mayor recorrido con pendientes moderadas, sus tractivo } \\
\text { son: Cerro E. D, quebradas, fauna variada y el Quetzal }\end{array}$ \\
\hline
\end{tabular}


de los recursos naturales de la reserva (Cuadro 5). En la Figura 4 se pueden observar las rutas de los diferentes senderos.

Los 7 senderos presentan características homogéneas en cuanto a: plantas epifitas; orquídeas de variadas formas y tamaños; bromelias; helechos pequeños y gigantes; musgos y abundante vegetación arbórea. La Fauna presente es
Análisis Espacial de Visibilidad de los Miradores. Cada sendero presenta una serie de puntos específicos, donde es posible observar paisajes hermosos y disfrutar de la flora y fauna. Esto evitar la monotonía en la observación de paisajes a los visitantes y reduce los costos de construcción de los miradores. El análisis de visibilidad se realiza desde un punto considerando
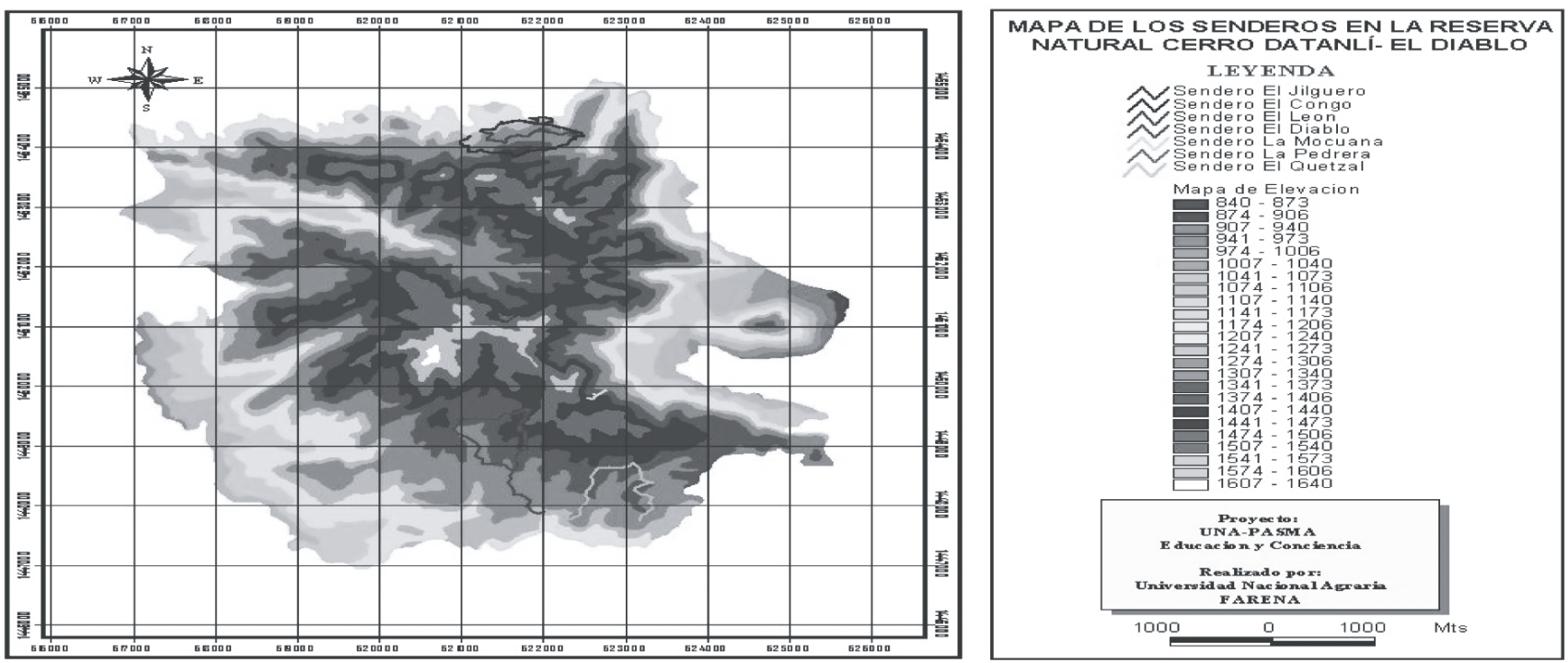

Figura 4. Mapa de los senderos de la zona norte y sur de la Reserva Natural Cerro "Datanlí-El Diablo". Jinotega, Nicaragua.

variada, sobresaliendo entre las aves el majestuoso Quetzal, así como El Rancho, El Jilguero, El Sargento y Tucanes; y entre las especies terrestres se encuentran Moluscos, Insectos, reptiles (Coral) y Mamíferos como Venados, Tigrillos, Guatusas, Sahino, etc. una altura de 2 metros sobre la superficie del suelo para determinar lo que es posible observar desde este punto.

En las figuras 5 y 6 se presentan un ejemplo de visibilidad desde un mirador y otro sobre la intersección de visibilidad desde dos miradores. En este sentido se recomienda realizar un estudio más detallado para plasmar
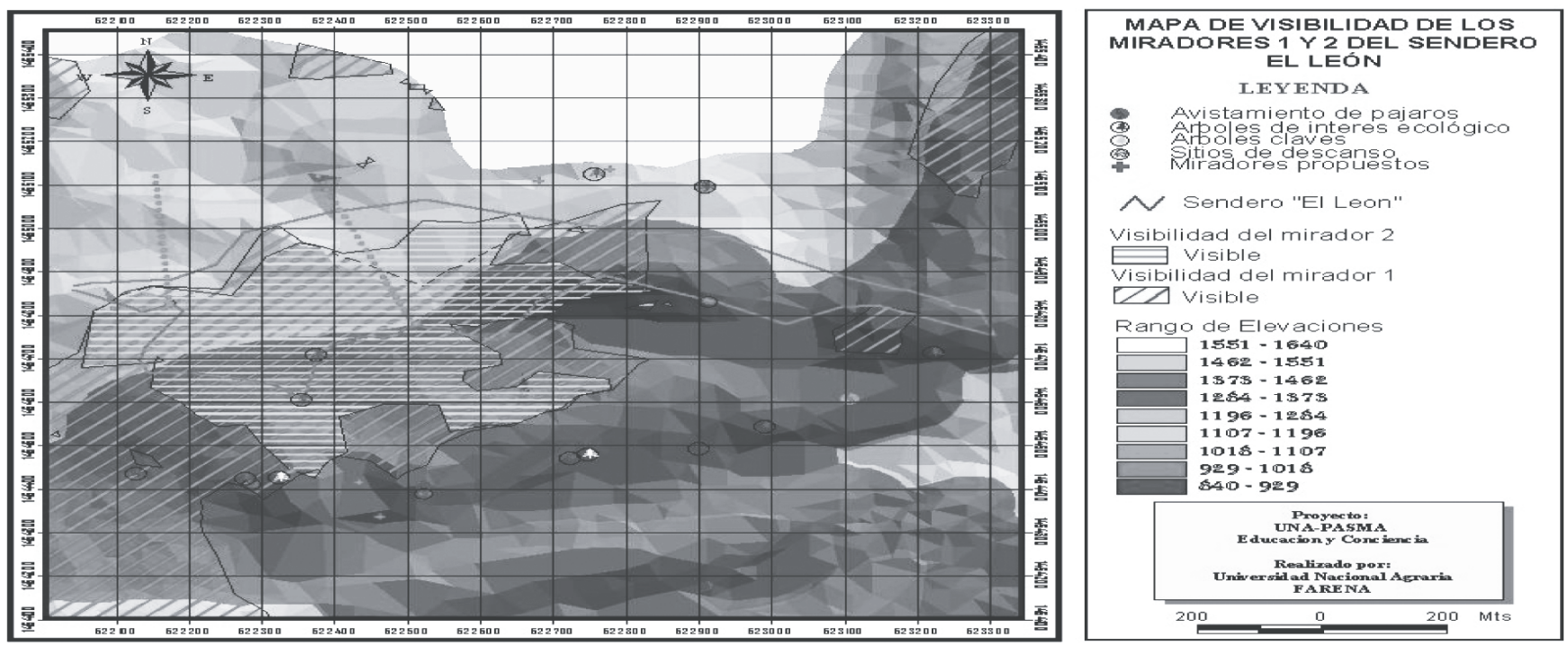

Figura 5. Ejemplo de análisis de visibilidad desde el mirador 1 y 2 del sendero El León en la zona norte de la Reserva Natural Cerro "Datanlí-El Diablo". Jinotega, Nicaragua. 
a través de toma fotográfica las bellezas que cada uno de ellos es capaz de ofrecer a los visitantes.

En vista a lo pronunciado de las pendientes se hace necesario modificar el recorrido en transeptos de los para realizar algún tipo de investigación que no dejan ni los documentos ni responden a estas necesidades básicas. De esta manera, no se podrá promover el turismo local o rural en la reserva. Promoción de Servicios y
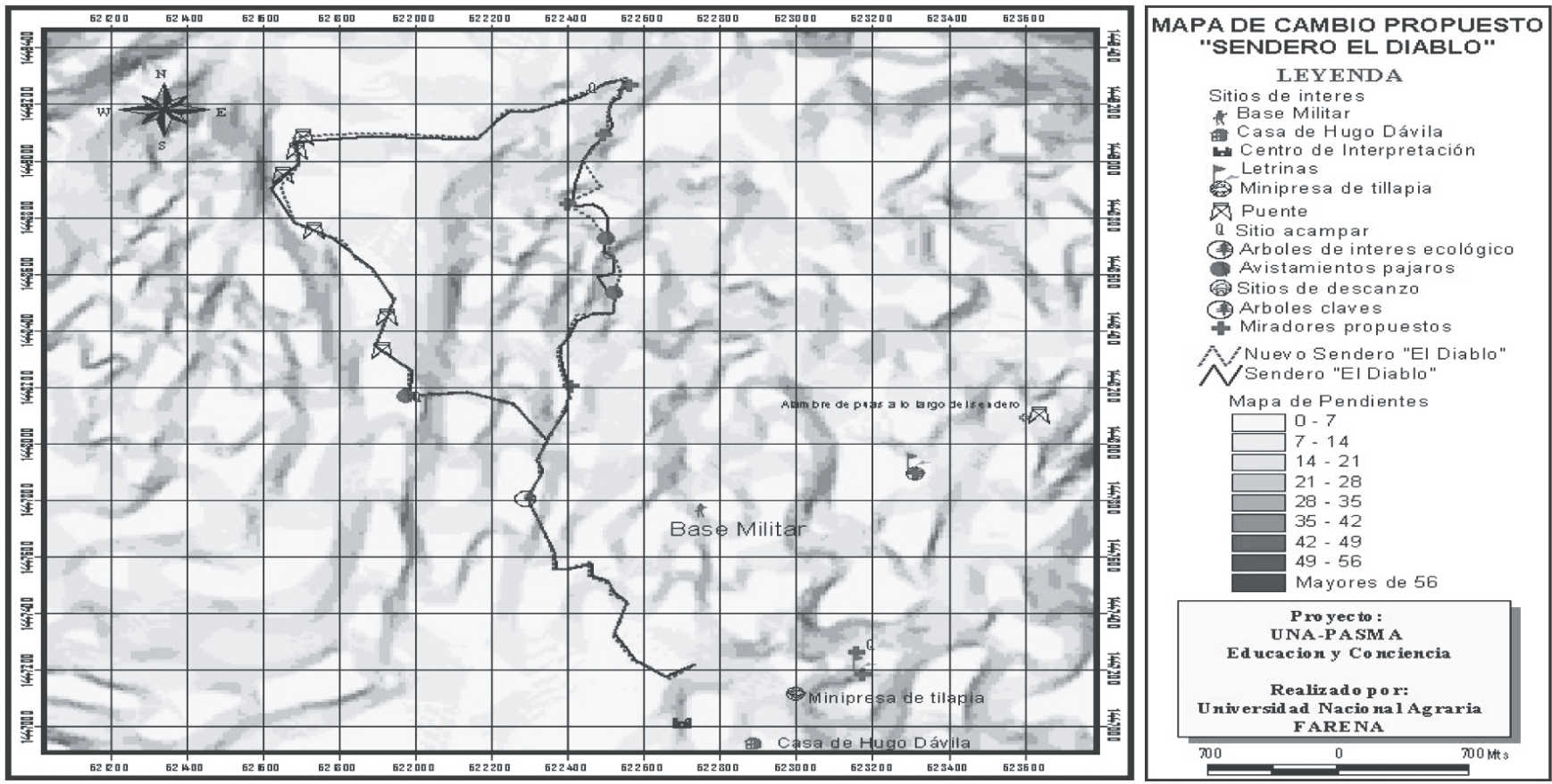

Figura 6. Cambios propuestos en el sendero El Diablo. RN Cerro Datanlí-El Diablo. Jinotega.

senderos y se proponen establecer algunos sitios para acampar, puestos de basuras, diseño de gradas, miradores, centros de interpretación y puentes para el paso de los visitantes (ejemplo figura 6), ya que los senderos deben de contar con ciertas condiciones mínimas para el tráfico de los visitantes por diversas áreas en donde pueda existir algún peligro de accidentes, es decir los caminos no permitan el paso, debido a ríos, quebradas o algunas zonas pantanosas.

Encuestas aplicadas a las variables Productores, Mujeres, Niños y Jóvenes Ambientalistas que viven en la Reserva.

Productores y mujeres. Percepción comunitaria, y Perfil ecoturístico: se concluye que los hombres consideran que el bosque, el potencial ecoturístico, los aspectos culturales y tradicionales en la reserva son valorados como excelentes; en cambio para las mujeres, la participación comunitaria en labores de conservación juega un papel fundamental, ya que la reserva tiene un alto potencial ecoturístico. Ambos coinciden que la falta de capacidad local administrativa (Luz, agua, caminos, infraestructura, etc), la toma de decisiones y el conocimiento en cuanto a los recursos naturales existentes en la reserva son sus mayores limitantes. Aunado a la falta de interés por parte de los proyectos que se han hecho presentes en la zona
Conservación: Los entrevistados respondieron que la reserva cuenta con diversidades de atractivos turísticos en cuento a paisajes, fuentes de agua, especies de flora y fauna de belleza e importancia ecológica y lugares desde donde se pueden observar sitios de gran interés para los visitantes nacionales y extranjeros.

A pesar de sus limitaciones, pueden ofrecer diversos tipos de servicios tales como trabajos manuales y/o artesanías (figura de animales hechos con el tallo de la cola de mono, hoyas de barro, canastos de carrizo, retratos de personas y maseteros de minas), Alimentos (guineo, tortillas, frijoles, malanga, refrescos naturales) y servicios de guías turísticas ( alquilar caballos, ofrecer paseos por los senderos), entre otros. Sin embargo, manifiestan que requieren la ayuda de proyectos en cuanto a capacitaciones encaminadas a esta finalidad, de lo contrario no podrán ofertar con calidad sus productos y además necesitan conocer donde se encuentran establecidos los senderos, ya que esta información solo la conocen los guarda parques de la zona, a pasar de que el área protegida es administrada de de manera conjunta. La producción y utilización de los recursos en la zona se hace con énfasis en el manejo sostenible y la conservación de los RRNN. Las Mujeres tienen una participación temporal en labores de huertos caseros y cocinando para los talleres que se imparten a los productores en la zona. Son integradas 
siempre y cuando estén organizadas en el programa de la Cuculmeca y sean parte del grupo de promotoras de salud. Expresaron de una manera literal que desean tener una participación conjunta en actividades de recreación, educación ambiental y en otros aspectos que tengan que ver con la reserva.

Niños y jóvenes ambientalistas. Cabe destacar que solo los niños de la zona Sur están bien organizados en las labores de conservación y protección de los recursos naturales de la reserva. Entre ellas figuran la no quema, reforestación, reciclaje de basura, no matar la fauna y otros. Sin embargo, en ambas zonas de una u otra forma los padres les han llevado a conocer algunos lugares de la reserva por lo que son conocedores del potencial de la misma y por ende sus apreciaciones indican que la valoran como excelente. Los guardaparques Harvey García y Sandro Fuentes de la zona Sur, son los encargados de brindarles capacitaciones a los niños de las escuelas Lázaro Talavera, El Paraíso y en el Instituto la Coculmeca.

Propuesta de tres oportunidades recreativas (paquetes turísticos) para su implementación en la Reserva.

Los paquetes que se proponen a continuación (pueden ser ofertados por una agencia o tour operadora o por los mismos actores locales, esto estará en dependencia del grado de organización y la capacitación del personal involucrado (comunidad, MARENA, PAGJINO, etc):

\section{Paquete No. 1. La Esmeralda}

Atractivo principal: Cascadas, Quetzales, Orquidiarios, Zoocriadero de Tilapias.

Actividades: Senderismo (Senderos El Quetzal, La Mocuana y La Pedrera), Contemplar paisajes, Tours en Caballos, visitas a los Orquidiario, Conocer Saltos de Agua, observar Quetzales, Visitar el Zoocriadero de Tilapia y Zoocriadero de Lombrices, Caza Fotográfica, Inmersión Sociocultural, Agro ecoturismo en Finca Las Carmelitas.

\section{Paquete No. 2. EI Diablo}

Atractivo principal: Quetzales, Cerro El Diablo, Saltos de Agua, Ojos de Agua, Bosque denso, Miradores.

Actividades: Senderismo, tours a caballo, Miradores de Fauna y Flora, cruzar por Quebradas, Contemplar paisajes, Caza Fotográfica, Leyenda Del Cerro El Diablo, observación de Quetzales, Jungle Tour.

\section{Paquete No. 3. Cerro El Gobiado}

Atractivos principales: Piedra del Amor, Peña del Gobiado, Bosque de Nebliselva Denso, Vista al Lago de Apanas a mas de 1, 300 msnm, Plantas Medicinales, Leyenda del Príncipe del Gobiado.

Actividades: Senderismo, Mirador de Aves

(Rancho, Quetzal y Jilguero), conocer Ojos de Aguas Curativos y Plantas Medicinales, Contemplar Paisajes, Tour a Caballos, Inmersión Social, vista de la Piedra del Amor, Peña del Gobiado, Leyenda del Príncipe del Gobiado, visitar la Estación Biológica de la UCA, Caza Fotográfica.

\section{CONCLUSIONES}

El pago por servicios ambientales, reparación y construcción de caminos de acceso y ausencia de organismos e instituciones son los principales problemas a resolver.

Se identificaron 22 principales atractivos turísticos (14 destacan aspectos de ecología de paisajes y 8 sitios que resaltan el valor histórico y algunas leyendas que tiene la reserva. Resultaron además, 17 sitios que pueden servir como miradores de paisajes y 5 sitios donde se pueden observar y escuchar cantar aves exóticas.

El traslape de visibilidad entre dos o mas miradores permitió hacer un análisis de las áreas que tienen aspectos en común y basados en estos resultados se propuso la eliminación o reubicación de estos con el fin de reducir costos al monumento del acondicionamiento de los senderos. 
La capacidad administrativa y gestión financiera, la toma de decisiones y conocimientos de los recursos naturales son muy pobres, por lo que son considerados como los obstáculos más importantes para el proceso de desarrollo del ecoturismo.

El área protegida es administrada por MARENA en coordinación con el Comité Local bajo un enfoque de manejo sostenible de sus RRNN que incluye talleres y capacitaciones para la conservación y protección de la reserva. Sin embargo, el $75 \%$ de las mujeres no son incluidas en éstas actividades.

La participación de los niños y jóvenes ambientalistas en actividades de conservación y protección de los recursos naturales de la reserva es más intensa y notable en la zona sur. Siendo una gran limitante para los niños de la zona norte. La ausencia de los servicios básicos (Luz y agua) así como el mal estado de los caminos son problemas que limitan el acceso a los visitantes.

Pese a que los paquetes turísticos son importantes para encaminar el desarrollo del ecoturismo en reservas naturales permitiendo divulgar y ofrecer los principales atractivos naturales de la reserva bajo un enfoque sostenible en el manejo de sus recursos naturales, es un proyecto que no a sido considerado para esta reserva.

\section{AGRADECIMIENTO}

Al proyecto UNA-PASMA/DANIDA, Educación y Conciencia por haber brindado el apoyo económico necesario para realizar todas las actividades respectivas y llevar a la elaboración final este trabajo de investigación. Y como mención principal a los productores Hugo Dávila y Ricardo Pineda, a los guardaparques Valerio Villegas, Severino Mairena, Sandro Fuentes y Harvey García.

\section{REFERENCIAS BIBLIOGRÁFICAS}

Astruc, J. 1996. Desarrollo del Ecoturismo en Rió San Juan. Presentación de la Primera Fase de Diagnostico. PNUD/ INTUR. Managua, Nicaragua.

Barzev, R. 1999. Valoración Económica del Potencial Turístico Isla de Ometepe. 17p.

FAO (Organización de las Naciones Unidas para la Agricultura y la Alimentación). 1993. Oltremari. El Turismo en los parques Nacionales y otras Áreas Protegidas de América Latina. 119 p.

GTZ-BMZ-UICN-WWF. 1999. Estándares para la evaluación y el monitoreo de la calidad del bosque a nivel de paisaje (Documento final) de Lammerts, B; Blom, E. 1997.

INTUR (Instituto Nicaragüense de Turismo, NI). 2002. Boletín de estadísticas de turismo 2002. Managua, Nicaragua. $69 \mathrm{p}$.

MARENA/PANIF APB. 1999. Biodiversidad en Nicaragua: un estudio de país. $1^{\text {ra }}$ edición. Managua, Nicaragua. $469 \mathrm{p}$.

MARENA (Ministerio del Ambiente y los Recursos Naturales, NI). 2002. Plan de Manejo de la Reserva Natural Cerro Datanlí-El Diablo. Resumen Ejecutivo. Jinotega, Nicaragua. 42 p.

MARENA (Ministerio del Ambiente y los Recursos Naturales, NI). 2002. Plan de Manejo Popularizado de la Reserva Natural Cerro Datanlí-El Diablo. Jinotega, Nicaragua. $35 \mathrm{p}$

MARENA (Ministerio del Ambiente y los Recursos Naturales, NI). 2004. Estado del Ambiente en Nicaragua 2003. II Informe GEO. Ministerio del Ambiente y los Recursos Naturales (MARENA), Segundo Proyecto de Desarrollo de Municipios Rurales (SPDMR-BM). Programa de Naciones Unidas para el Medio Ambiente, Oficina Regional para América Latina y el Caribe (PNUMA/ORPLAC).

Océano Práctico. 1999. Diccionario Océano Conciso de sinónimos y antónimos. Barcelona, España. Editorial Océano. $790 \mathrm{p}$.

Pagjino, 2003. Diagnóstico ambiental y posibles impactos producto de la propuesta de turismo de campo y ecoturismo en la Reserva Natural Cerro Datanlí-El Diablo. Jinotega, Nicaragua. 37 p.

Romero, E. 1996. El área de conservación llanuras de Tortuguero. Su paisaje y su Gente: una mirada introspectiva. $1^{\text {ra }}$. ed. San José. CR. 133 p.

UICN/OMT. 1993. Definición adoptada por la Unión Mundial para la Conservación Naturaleza y la Organización Mundial de Turismo del Arquitecto: Ceballos-Lascuráin. 